\title{
Electron bunch evolution in laser-wakefield acceleration
}

\author{
D. E. Cardenas, ${ }^{1,2,3}$ S. Chou, ${ }^{1,2,3,4}$ E. Wallin, ${ }^{3}$ J. Xu, ${ }^{1,5}$ L. Hofmann, ${ }^{1,2}$ A. Buck, ${ }^{1,2}$ K. Schmid, ${ }^{1,2}$ \\ D. E. Rivas, ${ }^{1,2,6}$ B. Shen, ${ }^{5}$ A. Gonoskov, ${ }^{7}$ M. Marklund, ${ }^{7}$ and L. Veisz ${ }^{1,3,{ }^{*}}$ \\ ${ }^{1}$ Max-Planck-Institut für Quantenoptik, Hans-Kopfermann Strasse 1, 85748, Garching, Germany \\ ${ }^{2}$ Ludwig-Maximilian-Universität München, Am Coulombwall 1, 85748, Garching, Germany \\ ${ }^{3}$ Department of Physics, Umeå University, SE-901 87 Umeå, Sweden \\ ${ }^{4}$ Center for High Energy and High Field Physics, National Central University, Chungli 32001, Taiwan \\ ${ }^{5}$ State Key Laboratory of High Field Laser Physics, Shangai Institute of Optics and Fine Mechanics, \\ Chinese Academy of Sciences, P.O. Box 800-211, Shangai, China \\ ${ }^{6}$ European X-Ray Free-Electron Laser Facility GmbH, Holzkoppel 4, 22869 Schenefeld, Germany \\ ${ }^{7}$ Department of Physics, University of Gothenburg, SE-41296 Gothenburg, Sweden
}

(Received 5 June 2020; accepted 4 November 2020; published 30 November 2020)

\begin{abstract}
We report on systematic and high-precision measurements of the evolution of electron beams in a laserwakefield accelerator (LWFA). Utilizing shock-front injection, a technique providing stable, tunable and high-quality electron bunches, acceleration and deceleration of few-MeV quasimonoenergetic beams were measured with cutting-edge technology sub-5-fs and 8-fs laser pulses. We explain the observations with dephasing, an effect that fundamentally limits the performance of LWFAs. Typical density dependent electron energy evolution with 57-300 $\mu \mathrm{m}$ dephasing length and 6-20 MeV peak energy was observed and is well described by a parabolic fit. This is a promising electron source for time-resolved few-fs electron diffraction.
\end{abstract}

DOI: 10.1103/PhysRevAccelBeams.23.112803

\section{INTRODUCTION}

Laser-wakefield acceleration (LWFA), a laser-driven electron acceleration scenario, was proposed in 1979 [1]. It was realized first in the hundreds of $\mathrm{MeV}$ energy range, [2-4] then at lower [5,6] as well as higher energies [7-9] generating multi-GeV beams. LWFA offers longitudinal accelerating fields on the order of $100 \mathrm{GV} / \mathrm{m}$, many orders of magnitude larger than conventional accelerators and it enables the generation of electron bunches with few-fs pulse duration $[10,11]$. Therefore, it provides compactness, novelty and accessibility for possible future applications. The maximum electron energy in a single accelerator stage is limited by laser diffraction, laser depletion and by the fact that the electrons are eventually faster than the laser-driven plasma wave. The latter is referred to as electron dephasing and the corresponding maximum acceleration length is the dephasing length $L_{d}$. It is given approximately by $L_{d, 3 D}=$ $\frac{4}{3} \sqrt{a_{0}} \lambda_{p}^{3} / \lambda_{0}^{2}$ for underdense plasmas in the $3 \mathrm{D}$ nonlinear theory [12] or by $\lambda_{p}^{3} / \lambda_{0}^{2}$ in a $1 \mathrm{D}$ weakly nonlinear scenario,

\footnotetext{
*Corresponding author. laszlo.veisz@umu.se

Published by the American Physical Society under the terms of the Creative Commons Attribution 4.0 International license. Further distribution of this work must maintain attribution to the author(s) and the published article's title, journal citation, and DOI. Funded by Bibsam.
}

where $\lambda_{p}[\mu \mathrm{m}]=3.3 \times 10^{10} / \sqrt{n_{e}\left[\mathrm{~cm}^{-3}\right]}$ and $\lambda_{0}$ are the plasma and laser wavelengths, respectively, and $n_{e}$ is the electron density. For acceleration lengths shorter than the dephasing length, the electron bunch always experiences an accelerating field and, thus, its energy increases. On the other hand, for acceleration lengths larger than the dephasing length, the bunch enters the region where the longitudinal accelerating field within the plasma period has changed sign and, therefore, it starts to decelerate. The energy increase continues until the longitudinal electric field reaches zero, at which point the acceleration length reaches $L_{d}$ and the maximum energy is obtained. In order to reach higher energies $>\mathrm{GeV}$, all energy limitations must be controlled in particular dephasing.

Various techniques have been implemented to mitigate diffraction such as preformed plasma channels [13], gasfilled capillary discharge waveguides $[9,14,15]$ or laser self-channeling [16]. In the high energy experiments cited above, in order to reach $\mathrm{GeV}$ range, a relatively low $\left(10^{17-18} \mathrm{~cm}^{-3}\right)$ electron density was used, thus electron dephasing was not the primary limiting factor. Instead, the laser was kept focused as long as possible, so that the electrons stayed in phase and were accelerated longer. Even if the electron dephasing is retarded by using low electron densities, there are still proposals to enlarge the dephasing length by using a tapered plasma medium with increasing density [17,18], plasma density tailoring [19], or a faster driver in a hybrid accelerator [20]. Recently, new concepts 
have even been proposed to circumvent the dephasing effect [21]. Therefore, better understanding and control of the maximum energy gain is fundamental to the physics of laser plasma accelerators.

In order to resonantly excite a plasma wave, the laser pulse length should match half of the plasma wavelength, i.e., $c \tau_{\text {pulse }}=\lambda_{p} / 2[1]$. Up to now, ultrashort $\approx 25 \mathrm{fs}$, highly intense (I $>10^{18} \mathrm{~W} \mathrm{~cm}^{-2}, a_{0}>1$, where $a_{0}$ is the normalized laser vector potential) Ti:sapphire laser pulses $\left(\lambda_{0}=800 \mathrm{~nm}\right)$ were typically employed, which led to dephasing lengths of many mm's. As $L_{d} \propto \lambda_{p}^{3} \propto \tau_{\text {pulse }}^{3}[6]$, shorter pulses, matched densities and relativistic intensities lead to dephasing effects within $10 \mathrm{~s} \mu \mathrm{m}$. Optical parametric chirped pulse amplification (OPCPA) [22] technology allows the production of multi-TW sub-10-fs laser pulses as demonstrated by the Light Wave Synthesizer 20 (LWS20). The upgraded sub-5-fs [23] (older 8-fs [24]) version of LWS-20 is (was) the most intense few-cycle laser system in the world.

A possible application of the electron bunches is ultrafast electron diffraction (UED). Traditional UED utilizing an electron gun $[25,26]$ is an effective tabletop technique to study atomic motions in matter with femtosecond temporal

(a)

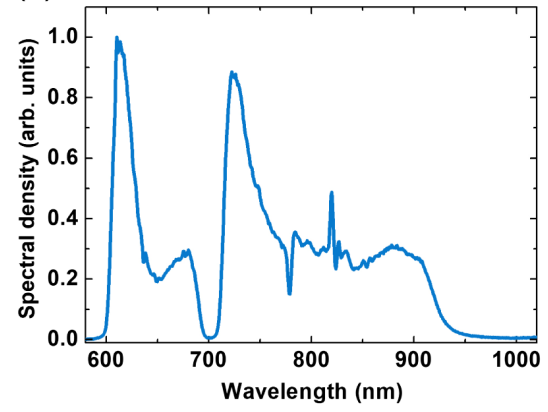

resolution. The resolution of UED is determined by the duration of the electron bunches and the time-of-arrival jitter between these bunches and a laser pulse. A recent development is the application of relativistic electron pulses to suppress space charge effects that degrade the electron bunch duration [27]. Typical main parameters of state-ofthe-art MeV UED facilities [28,29] are 2.5-6 MeV electron energy with about $0.1 \%$ energy spread, $1-10 \mathrm{fC}$ electron bunch charge, $20-50 \mu \mathrm{rad}$ divergence, about $20 \mathrm{~nm}$ transverse normalized emittance, and 50 fs FWHM temporal resolution at best [30].

In this article, we present the first systematic measurement of the electron beam evolution in a LWFA. The short sub-10-fs laser pulses give rise to acceleration to an electron energy of up to $10-20 \mathrm{MeV}$, and a dephasing within about 57-300 $\mu \mathrm{m}$. To reveal electron beam evolution, the acceleration length is scanned by varying the position of electron injection into the plasma wave. The injection mechanism used in this experiment is the so-called shock-front injection [31]. It has proven to work for long pulses ( $\approx 25 \mathrm{fs})$ [19, 32-34] as well as for short ones [31] providing stable, highquality, quasimonoenergetic electron beams with low energy spread $\left(\Delta E_{\mathrm{FWHM}}<5 \mathrm{MeV}\right)$ and tunability of the

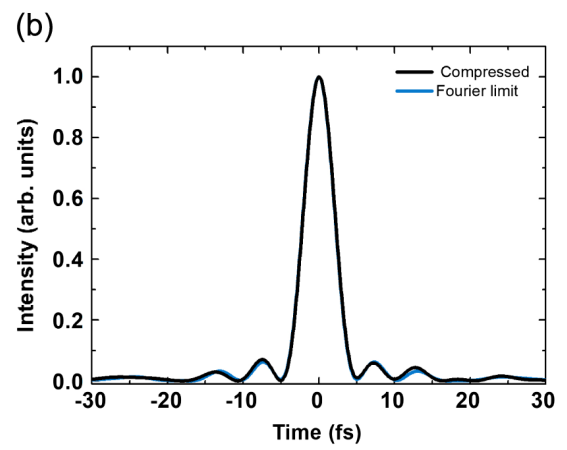

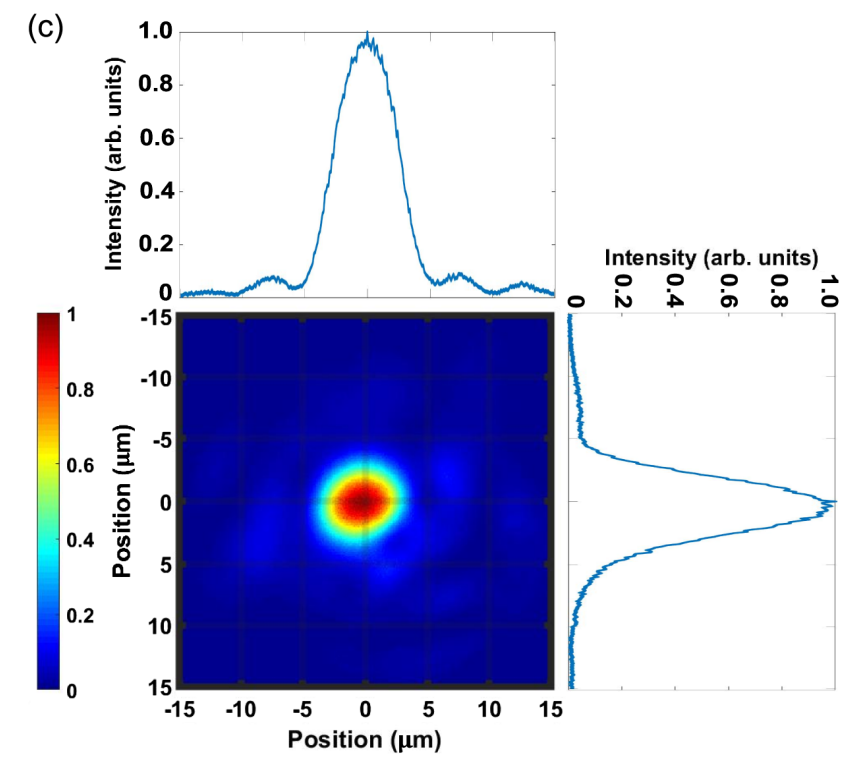

FIG. 1. Typical laser parameters of the $<5$-fs system. (a) Spectrum of the LWS-20; (b) compressed (4.53 fs) and Fourier limited temporal intensity profile; (c) LWS-20 focal spot profile using an off-axis parabola with $f \#=3.3$. 
electron spectrum over more than 1 order of magnitude by using a long laser, as in [32]. Shock-front injection is realized by placing a razor blade or silicon wafer on top of a supersonic gas nozzle, creating an ultrathin $(<5 \mu \mathrm{m})$ shock front which separates two different plasma regions with a certain density ratio $\left(\approx 1.6\right.$ in our case $[31], n_{e, 1}>n_{e, 2}$; $\lambda_{p, 1}<\lambda_{p, 2}$ in case of a down-ramp transition). As the laser passes the density shift, a fraction of the background plasma electrons, forming the end of the first plasma period in $n_{e, 1}$, is injected. These electrons are inserted in the accelerating phase of the first plasma period in the lower density range $n_{e, 2}$. In this configuration, such a sharp density transition allows an instantaneous and precise injection and therefore, very low and peak-energy-independent (constant) absolute energy spread of the electron beam. This simple but effective method provides high control over the electron injection position and thus over the final peak energy, by shifting the blade and correspondingly the shock-front position.

\section{EXPERIMENT}

In the first part of our experiments, we focused the old version of LWS-20 with $8 \mathrm{fs}, 800 \mathrm{~nm}$ central wavelength, $65 \mathrm{~mJ}$ on-target energy pulses $(130 \mathrm{~mJ}$ at the end of the laser) to a spot of $12 \mu \mathrm{m}$ (FWHM) with $f \#=6$, reaching a peak intensity of $1.6 \times 10^{18} \mathrm{~W} \mathrm{~cm}^{-2}$ [31]. In the second part, we used the upgraded LWS-20 (see Fig. 1) with $<5$ fs, $740 \mathrm{~nm}, 37 \mathrm{~mJ}$ on-target energy pulses $(75 \mathrm{~mJ}$ at the end of the laser) focused to a spot of $5.5 \mu \mathrm{m}$ (FWHM) with $f \#=3.3$ obtaining a peak intensity of $1.2 \times 10^{19} \mathrm{~W} \mathrm{~cm}^{-2}$ (which was higher than the applied input peak intensity due to prechirping the pulses as described later).

A fully characterized $300 \mu \mathrm{m}$, for the $<5$-fs case, (500 $\mu \mathrm{m}$ for the 8 -fs case) supersonic de Laval nozzle

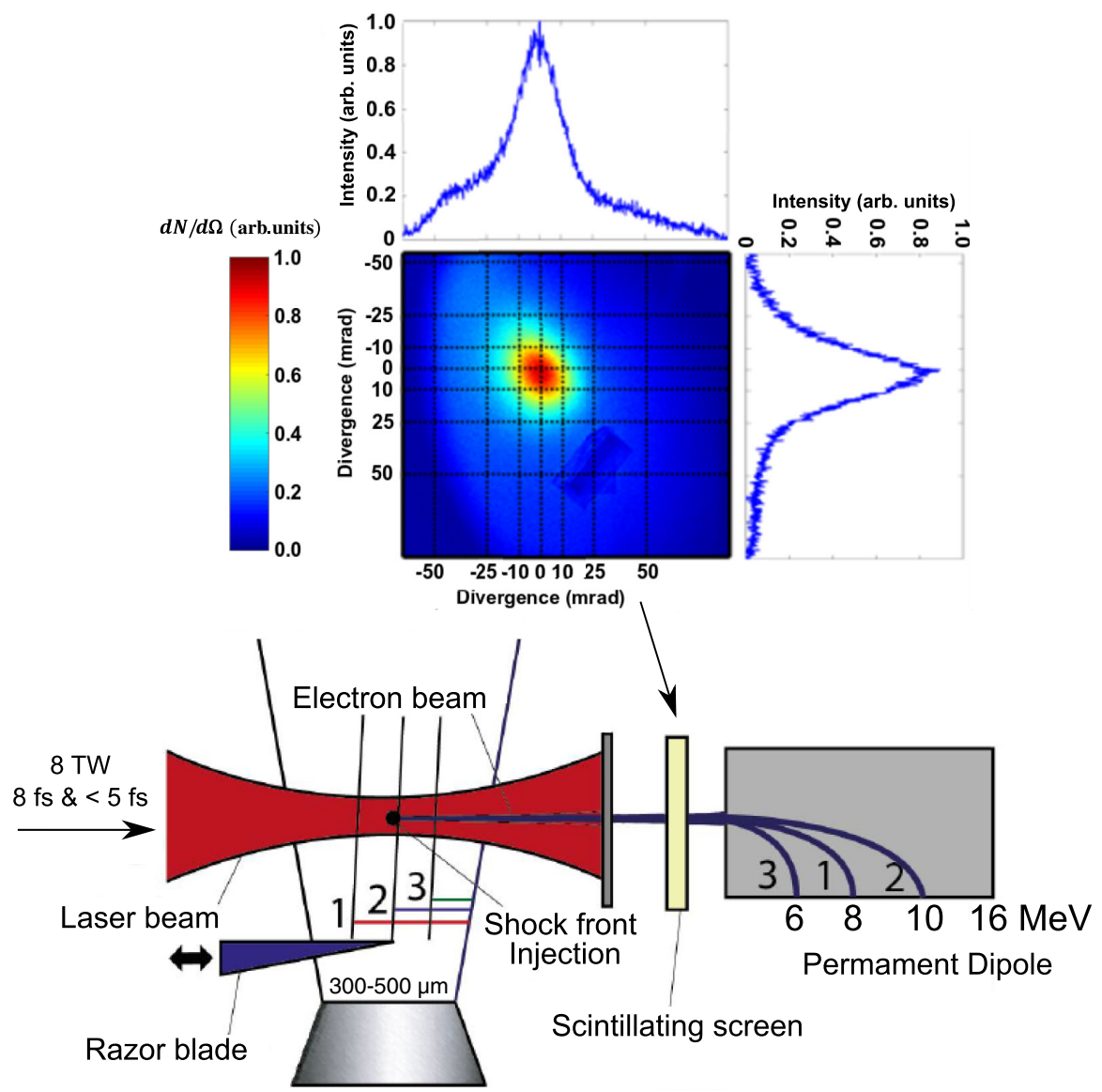

FIG. 2. Experimental setup: The laser beam was focused on helium gas emanating from a $300 \mu \mathrm{m}(<5 \mathrm{fs})$ and $500 \mu \mathrm{m}(8 \mathrm{fs})$ supersonic de Laval nozzle. A razor blade produced a sharp down-ramp density transition with a density ratio 1.6, the so-called shock front. The blade was positioned $100 \mu \mathrm{m}$ above the nozzle and 100-200 $\mu \mathrm{m}$ below the laser and was moved longitudinally to tune the acceleration length of the injected electron bunch. A removable scintillating screen (BIOMAX) was used for absolute charge measurement and beam profile observation. A permanent dipole magnet with entrance aperture, equipped with BIOMAX as well, was installed to measure the electron spectrum. A probe beam propagated through the nozzle perpendicularly to the main beam. Different injection positions are illustrated corresponding to different acceleration lengths: longer (1), similar (2) and shorter (3) than the dephasing length. Typical electron spectra from (1), (2) and (3) are shown in Fig. 3. Inset: Typical electron beam profile in the sub-5-fs case: $2 \mathrm{pC}$ charge and 10-mrad root-mean-square (rms) divergence. 


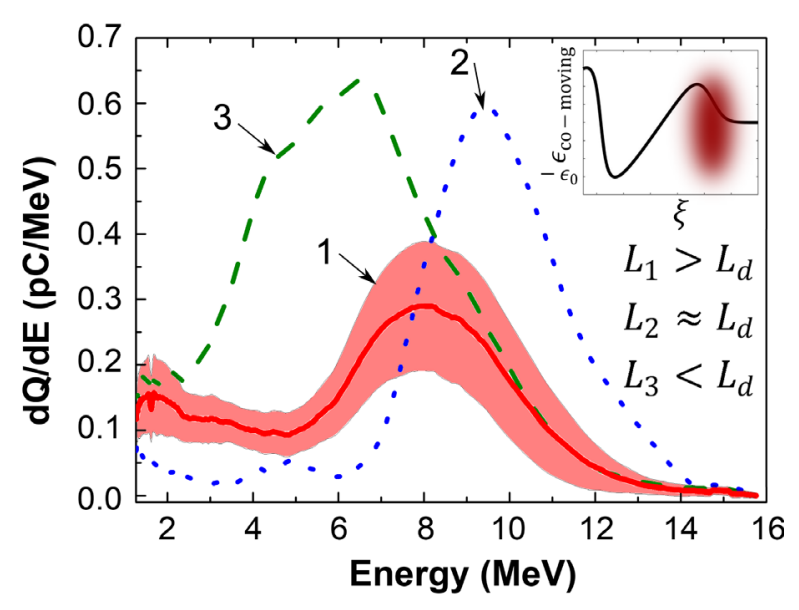

FIG. 3. Average electron spectrum with $8 \mathrm{MeV}$ peak energy over 20 shots (for $L_{1}$ ) and standard deviation band in the shaded area, and two typical single-shot electron spectra (for $L_{2}$ and $L_{3}$ ) at $7.7 \times 10^{19} \mathrm{~cm}^{-3}$ and $<5$-fs LWS-20 pulses with peak energies (centroid of a Gaussian fit after background subtraction) of 9.5 and $6 \mathrm{MeV}$, respectively. The measured dephasing length is $L_{d} \approx 130 \mu \mathrm{m}$ (see Fig. 6). Inset: Longitudinal electric field of the plasma in the comoving frame inside the first plasma period.

with helium gas and a razor blade on a translation stage provided the acceleration medium with controlled injection, as seen in Fig. 2. The background electron density was tuned within the range of $4-21 \times 10^{19} \mathrm{~cm}^{-3}$ to approximately match the corresponding laser pulse duration. Shadowgraphy with a probe beam propagating perpendicularly to the main beam was used to observe the plasma channel, the plasma wave and the injection position of the electrons. The plasma wavelength, i.e., the electron density, is matched to the laser pulse duration in a pure LWFA scenario. However, within a small range of densities away from this resonance, wakefield excitation and electron acceleration also take place. This enabled us to measure the electron energy evolution in more than two cases, even though only two different pulse durations were employed. Furthermore, although instabilities in the accelerator are expected at higher densities in the self-injection regime [35], these are not present for the external injection mechanism used in this work. While scanning the injection position, the focus of the laser was moved accordingly in order to compensate the laser diffraction. For each injection position, especially in the sub-5-fs case, chirping the laser pulse about +10 to $+20 \mathrm{fs}^{2}$ before the interaction helped to compensate the dispersion in the plasma $\left(-7 \mathrm{fs}^{2} / 100 \mu \mathrm{m}\right.$ at $\left.1 \times 10^{20} \mathrm{~cm}^{-3}\right)$. The input peak intensity was thus lower $(\approx 1 / 2)$, i.e., $\approx 4-6 \times 10^{18} \mathrm{~W} \mathrm{~cm}^{-2}$ but remained more stable throughout the acceleration medium.

For the 8-fs case, typical electron bunches had approximately $8 \mathrm{pC}$ charge, peak energies of 5-20 MeV and approximately $4 \mathrm{mrad}$ rms divergence. While for the sub5 -fs case, $2 \mathrm{pC}$ [36] bunches with rather large divergence (10 mrad rms) were generated at $<10 \mathrm{MeV}$ peak energies. Due to the divergence of the electron beam with our typical energy spread, peak energies below 2-3 MeV cannot be measured properly and low $(5 \mathrm{MeV})$ as well as medium $(12 \mathrm{MeV})$ energies have a maximum central energy shift of $5 \%$. The electron spectra are plotted for different acceleration lengths in Fig. 3 using sub-5-fs laser pulses. The acceleration length is determined from the relative injection position [see Fig. 4(a)] with the help of a parabolic fit as described later. The electron energy increased with acceleration length $L_{3}$, reached its maximum at $L_{2}$ and decreased at $L_{1}$. The acceleration lengths were scanned

\section{(a)}

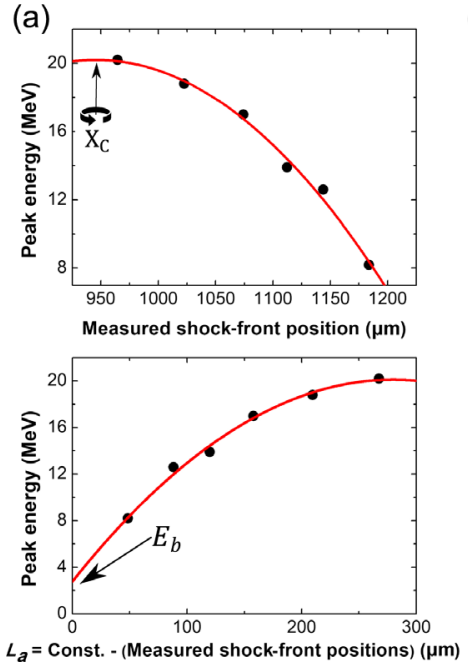

(b)

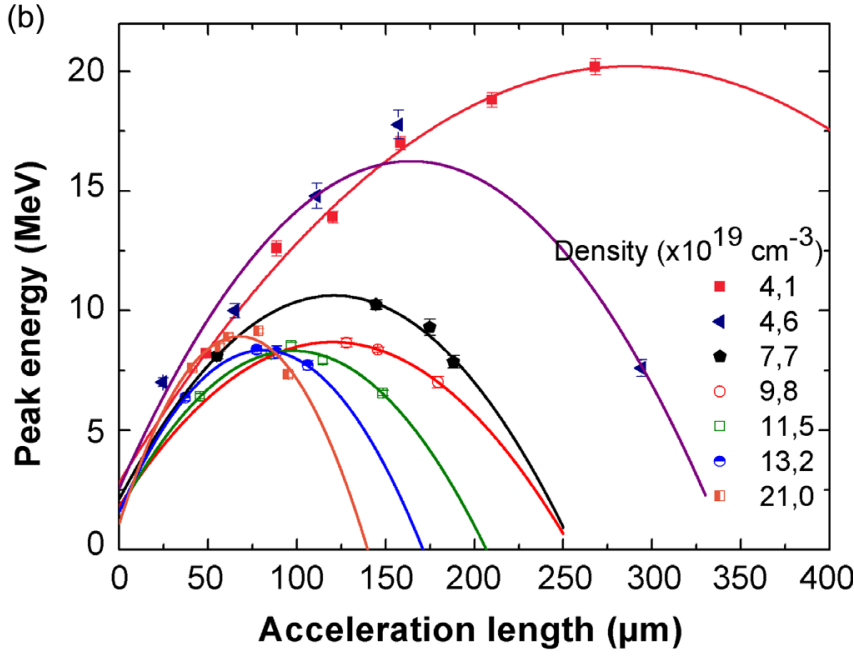

FIG. 4. (a) Retrieval of acceleration lengths $L_{a}$ from the measured shock-front positions (see details in text). (b) Peak energy of the electron spectrum for 8 -fs $\left(<5 \times 10^{19} \mathrm{~cm}^{-3}\right)$ and sub-5-fs (all others) laser pulses for different acceleration lengths for various electron densities. The vertical error bars indicate the standard error over 50 shots whereas the horizontal error bar is approximately $5 \mu$ m, which is comparable to the symbol size and therefore not plotted. The lines are fits using Eq. (2). 


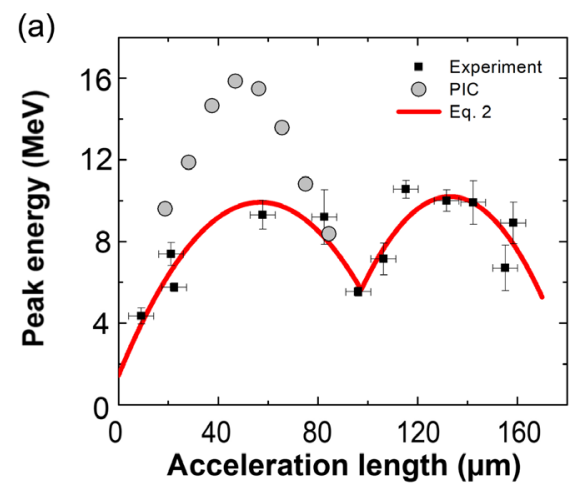

(c)

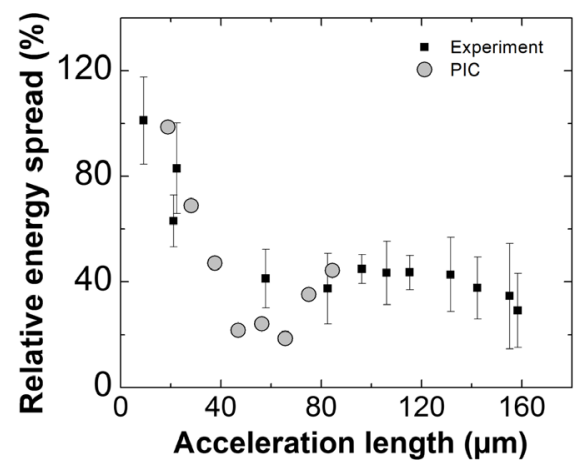

(b)

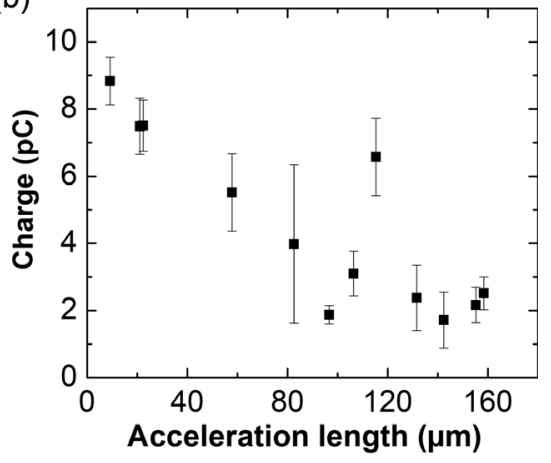

(d)

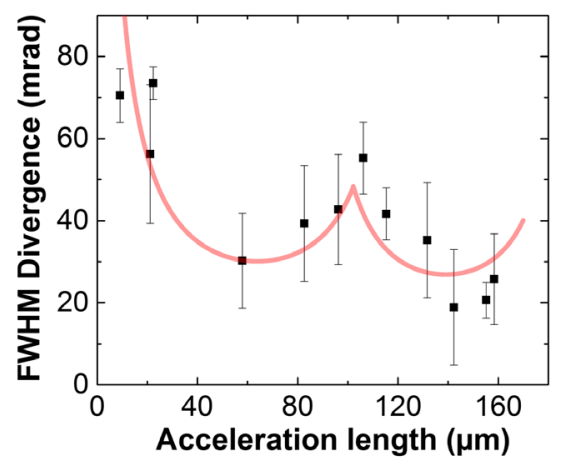

FIG. 5. Evolution of the electron bunch at $14.4 \times 10^{19} \mathrm{~cm}^{-3}$ driven by sub-5-fs laser pulses for 12 different injection positions. (a) Peak energy of the measured (black squares) and PIC simulated (gray filled circles) electron spectra, and parabolic fit (continuous red line) from Eq. (2); (b) electron bunch charge; (c) measured (black squares) and PIC simulated (gray filled circles) relative energy spread; (d) beam divergence (black squares) and 635/ $\gamma_{e}$ mrad fit (continuous line) from Eq. (2) as a function of the acceleration length. Error bars correspond to the standard deviation of about 50 shots per injection position. Horizontal error bars from (a) apply as well to (b)-(d).

in a range large enough for the dephasing effect to manifest. This procedure was repeated for electron densities of $4.1 \times$ $10^{19} \mathrm{~cm}^{-3}$ and $4.6 \times 10^{19} \mathrm{~cm}^{-3}$ for the older 8-fs LWS-20 and from $7.7 \times 10^{19} \mathrm{~cm}^{-3}$ up to $21 \times 10^{19} \mathrm{~cm}^{-3}$ with the $<5$-fs system. The observed peak energies are plotted as a function of the acceleration lengths in Fig. 4(b).

We observed in many cases that for long enough acceleration lengths, $L_{a}>L_{d}$, the peak energy of the electron beam increased again, as plotted in Fig. 5(a) for $14.4 \times 10^{19} \mathrm{~cm}^{-3}$, in contrary to the results in [37]. The charge continuously decreased along the whole acceleration length [see Fig. 5(b)] with the slope of $-0.8 \mathrm{pC} / 10 \mu \mathrm{m}$ up to $L_{a} \approx 100 \mu \mathrm{m}$ and later remained constant. The absolute energy spread stayed constant $(\approx 3.5 \mathrm{MeV})$ up to the first dephasing point $L_{a} \approx 60 \mu \mathrm{m}$ due to the injection technique [32]. Consequently, the relative energy spread improved by almost a factor of 3 , proportional to the energy gain, and afterwards it remained approximately constant [see Fig. 5(c)]. Finally, the divergence of the electron bunch behaves as $1 / \gamma_{e}$ throughout the whole process, where $\gamma_{e}$ is the gamma factor of the electron bunch [see Fig. 5(d)]. Moreover, the spatial profile of the accelerated beams remains roughly circular for all acceleration lengths indicating a very weak interaction of the electrons with the laser during the acceleration [38], in particular at the dephasing region.

\section{ANALYSIS AND DISCUSSION}

Through parabolic fitting of the experimental data, as shown in Fig. 4(b), we obtained the dephasing length as well as the accelerating fields in each case. The analysis and the retrieval of the results are expected to work under the following approximations.

(i) The longitudinal accelerating field $\epsilon_{\text {comoving }}$ scales linearly with the longitudinal coordinate $\xi$ in the comoving frame, which is a characteristic feature of nonlinear plasma waves (see the inset in Fig. 3). Furthermore, this linear dependence is also valid within the laboratory frame, which is reasonable for constant speed difference between electrons and plasma wave. This forms the basis of the fit as it will be shown.

(ii) The whole wakefield generation takes place in a flattop electron density profile, where effects from plasma gradients at the edges are insignificant.

(iii) The parabolic fitting is based on a 1D-like weakly nonlinear plasma wave [39] and further relativistic effects generated by the laser are not taken into account. Such a scenario fits well to shock-front injection [31,32]. 
(iv) Any dynamical changes of the first plasma period, referred as bubble, or variations in the group velocity of the laser are not included. Thus, it does not cover the whole physical picture $[12,40-43]$ and rather assumes a constant accelerating field.

(v) Beamloading is not significant, as shortly discussed later.

(vi) Electron beam interactions with the laser are not significant. In a more realistic case, the laser pulse is shorter than half a plasma period $c \tau_{L} \leq \lambda_{p} / 2$, due to self-compression in plasma [44]. Therefore, this condition is fulfilled at least until the dephasing point even for the strongly nonlinear blowout case.

The longitudinal electric field of the plasma at the position of the electron bunch $\epsilon$ as a function of its position in the laboratory system $x$ is

$$
\epsilon(x)=\epsilon_{0}-\frac{\epsilon_{0}}{L_{d}} x ; \quad 0 \leq x \leq 2 L_{d},
$$

where $\epsilon_{0}$ is the longitudinal accelerating field at the rear of the first plasma period (see inset in Fig. 3). The accelerating field is zero at the position where the dephasing limit is reached. The electron energy is obtained by adding to the initial kinetic energy the integral of the field Eq. (1) from the injection position $x=0$ up to a certain acceleration length $L_{a}$. We assume that the initial kinetic energy of a trapped electron in the plasma wave matches the linear group velocity of the laser and it is determined by $E_{b}=$ $0.511 \mathrm{MeV}\left(\lambda_{p} / \lambda_{0}-1\right)$. This yields

$E_{p}\left(L_{a}\right)=\int_{0}^{L_{a}} e \epsilon(x) d x+E_{b}=e L_{a} \epsilon_{0}\left(1-\frac{L_{a}}{2 L_{d}}\right)+E_{b}$.

The peak energy of the electron spectrum $E_{p}$ depends quadratically on the acceleration length $L_{a}$. In a real scenario, $L_{a}$ is not directly measured due to the gradient of the plasma profile. Nevertheless, only the relative injection positions into the wakefield determine the fit. From the fit coefficients, the accelerating field as well as the dephasing length are retrievable. For lower densities, $e L_{d} E_{a, 0} \gg E_{b}$ applies. However, in this highly dense regime, the phase velocity of the plasma is also not negligible $\left(E_{b} \approx 1-3 \mathrm{MeV}\right)$ compared to the maximum obtained energy (8-20 MeV) and therefore this last term on the right side of Eq. (2) is not negligible. Figure 4(b) shows fits given by Eq. (2) to the measured electron peak energies as functions of the acceleration length for different densities. In the few cases where the spectra are not Gaussian-like, we used the first moment of the spectral distribution. Our investigations show that this definition is much more accurate than taking the high-energy cutoff of a continuous energy distribution $[37,45]$. These fits and the acceleration length were obtained in the following way [see Fig. 4(a)].

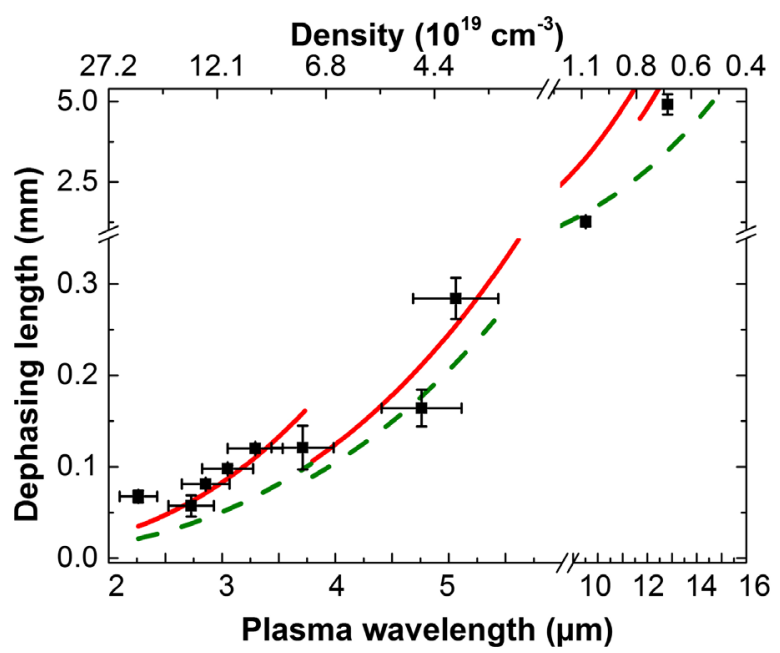

FIG. 6. Measured (black squares) and predicted dephasing lengths according to $L_{d_{3 D}}$ (continuous red line [12]) using $a_{0,5 \mathrm{fs}+\mathrm{CHIRP}}=1.5$ and $a_{0,8 \mathrm{fs}}=0.8$; and $1 \mathrm{D}$ weakly nonlinear theory (dashed green line). The result at $\lambda_{p} \approx 9.5 \mu \mathrm{m}$ is from [37] with an $a_{0}$ of 2.5, while at $\lambda_{p} \approx 12.7 \mu \mathrm{m}$ is from [45] with an $a_{0}$ of 2.9. Vertical error bars are the standard deviation from the fits while horizontal error bars correspond to $15 \%$ uncertainty in the electron density determination.

(i) Plot the peak energy determined from the (averaged) spectrum with respect to the relative injection positions, measured directly from the plasma channel observation. Increasing injection positions correspond to decreasing acceleration lengths.

(ii) Fit a parabola to this plot via the least-squares method and mirror (invert) it and the measurement points horizontally with respect to the center of the parabola. Shift the parabola along the (horizontal) acceleration length axis until the intersection with the peak energy axis matches $E_{b}$. The acceleration length plotted in Fig. 4(b) is then the distance between the $x=0$ and the inverted and shifted injection positions. Since $E_{b}$ is calculated directly from the electron density measurements, the fit has only two degrees of freedom. The corresponding $L_{a}$ is a constant minus the injection position.

Figure 6 shows the retrieved $L_{d}$ from the fits using Eq. (2) in Fig. 4(b). Even though for the longest acceleration lengths extinction of the wakefield or nonlinearities may affect the acceleration process, the energy evolution seems to be mainly limited by the dephasing of the electron beam. Our results match very well the $1 \mathrm{D}$ weakly nonlinear dephasing formula $\lambda_{p}^{3} / \lambda_{0}^{2}$. They also fit the $3 \mathrm{D}$ nonlinear formula shown in the introduction since in a weakly relativistic regime both expressions predict similar results, as visible in Fig. 6. For the sub-5-fs case, a lower $a_{0}$ is used in the 3D formula due to the experimentally applied initial chirp. For the sake of completeness and to validate our analysis at other densities and laser parameters, we plot the results retrieved from the experiments from $[37,45]$. 
The very good agreement with theory confirms that our simple but consistent analysis describes dephasing well and laser propagation does not influence significantly our high density results as changes of the laser pulse in these studies $[37,45]$, at lower densities, are much less relevant compared to our parameters. Particularly, the result from [37] was identified as longitudinally self-injected electrons. In this regime, nonlinearities are not significant, as stated by the authors. Moreover, the fact that the fit also describes correctly the results from other groups, where different gas targets were utilized, supports our claim that potential effects arising from plasma density modulations or gradients are not significant. Furthermore, in our case $\frac{P}{P_{c r}}<3$ initially for both laser systems, where $P_{c r}$ is the critical power for relativistic self-focusing [46], except for at $n_{e}=$ $2.1 \times 10^{20} \mathrm{~cm}^{-3}$ where the retrieved $L_{d}$ deviates significantly from the expected value. Short dephasing lengths (57-300 $\mu \mathrm{m})$ are a direct consequence of employing highly dense plasmas and sub-10-fs pulses. Moreover, these results clearly indicate that in order to reach high energy electrons, longer pulses and lower electron densities should be applied to excite the wakefield, i.e., $E_{p, \max } \propto$ $e \epsilon_{0} L_{d} \propto \lambda_{p}^{2}$, as observed using these two laser systems, $20 \mathrm{MeV} / 8 \mathrm{MeV} \approx\left(\frac{5 \mu \mathrm{m}}{3 \mu \mathrm{m}}\right)^{2}$ in Fig. 4(b). Furthermore, we present two experimental reasons to support the fact dephasing has limited the electron energy: (i) the laser energy depletion was measured to be $10 \%-20 \%$, at the most, after the interaction; (ii) all the acceleration lengths considered to plot the fits are shorter than the measured confocal length $\left(2 Z_{R} \approx 240 \mu \mathrm{m}\right.$ in the sub-5-fs case). These previous observations allow us to claim that neither diffraction nor depletion played a significant role. Moreover, no self-injection was observed when the blade was removed. The inefficient laser energy coupling to the plasma, along with the observation of only blueshift in the transmitted laser spectrum, further contribute to understand that our experiments were not in the strongly nonlinear regime [44] but rather in a weakly nonlinear scenario. Corrections to the wake phase velocity as studied in [43] would represent a maximum deviation of 5\% from the current dephasing length results. Therefore, the linear laser group velocity is a good approximation. The longitudinal accelerating fields from the fit were on the order of $100-250 \mathrm{GV} / \mathrm{m}$. The values were smaller than the theoretical values of up to $1 \mathrm{TV} / \mathrm{m}$ due to our modest intensities $a_{0} \approx 1$, far away from the complete blowout $a_{0} \geq 4$ [47-49]. Furthermore, low energy coupling into the plasma hinders charge separation during the plasma wave excitation resulting in a low accelerating field, as measured. Beamloading was recognized [50] but turned out only to have an effect of $20 \%$, at the most, in the electron peak energy and almost no effect in the absolute energy spread.

\section{SIMULATIONS}

3D particle-in-cell (PIC) simulations with a 5-fs driver laser were performed using the code ELMIS3D [51] and VORPAL [52] code for the 8-fs driver to investigate details of the dephasing process and post-reacceleration. The simulation boxes were $30 \times 30 \times 30 \mu \mathrm{m}^{3}$ and $20 \times 30 \times$ $30 \mu \mathrm{m}^{3}$ for the 5 -fs and the 8-fs cases, respectively; and moved at the speed of light. They were divided into $512 \times$ $256 \times 256$ and $400 \times 300 \times 300$ cells, each one containing 20 and 1 macroparticle inside for the 5 -fs and the 8 -fs cases, respectively. In the simulations the electrons are not exactly trapped at the injection position at the shock front. After the sharp down-ramp transition (from $1.1 \times 10^{20} \mathrm{~cm}^{-3}$ to $7 \times$ $10^{19} \mathrm{~cm}^{-3}$ within $<3 \mu \mathrm{m}$ ) and intense dynamics in the first few $\mu$ m's, a significant portion of the electrons leave the first period and most of the particles that will be trapped fall back almost to the end of the first plasma period similarly to the case in [31]. Therefore, the trapping process does not shorten significantly the dephasing length. Moreover, the location of the shock front is $100 \mu \mathrm{m}$ in front of the focal plane "in vacuum" resembling the experimental conditions, which contribute to a lower, but more stable on-axis laser intensity along the acceleration. The simulated results agree qualitatively with the experimental observations and yield similar dephasing lengths: $\approx 50 \mu \mathrm{m}$ for $n_{e}=$ $7 \times 10^{19} \mathrm{~cm}^{-3}$ and 5 -fs pulses and $\approx 130 \mu \mathrm{m}$ for $n_{e}=$ $4 \times 10^{19} \mathrm{~cm}^{-3}$ and 8-fs pulses. In the 8-fs simulations, we observed an improvement of the relative energy spread up to $L_{a} \approx L_{d}$. The wakefield in the latter case is stable for at least $200 \mu \mathrm{m}$. The simulations with the 5-fs driver show two regimes: (1) one in which a single bunch dephases in a classical scenario (Fig. 7); and (2) an extreme case where a second bunch is self-injected and accelerated in the plasma wakefield driven by the original bunch [53]. For the normal dephasing case (1), the absolute energy spread remains constant until the dephasing point and similar parabolas are obtained for the energy vs acceleration length as experimentally observed. In Fig. 7(a) we can even see the start of the acceleration of a weak second electron bunch at around $45 \mu \mathrm{m}$, which signals the start of the plasma wakefield. Although, the plasma wakefield case (2) is beyond the scope of this paper, this scenario is only observed when the driver is powerful enough (x1.5 higher energy) and initially chirped to compensate the plasma dispersion. This exotic case resembles the "double parabola" energy evolution shown in Fig. 5(a). Most importantly, the dephasing length retrieved from the first parabola is almost identical to the corresponding one in Fig. 7. In the experiments, an extra "on-target" optimized wave front and correspondingly $35 \%-40 \%$ higher energy content of the focus, and therefore higher peak intensity, led to the observations of this rare regime in $25 \%$ of the cases. The above numerical results allow us to claim that dephasing was reached in all cases and other effects, such as depletion, diffraction, or laser 

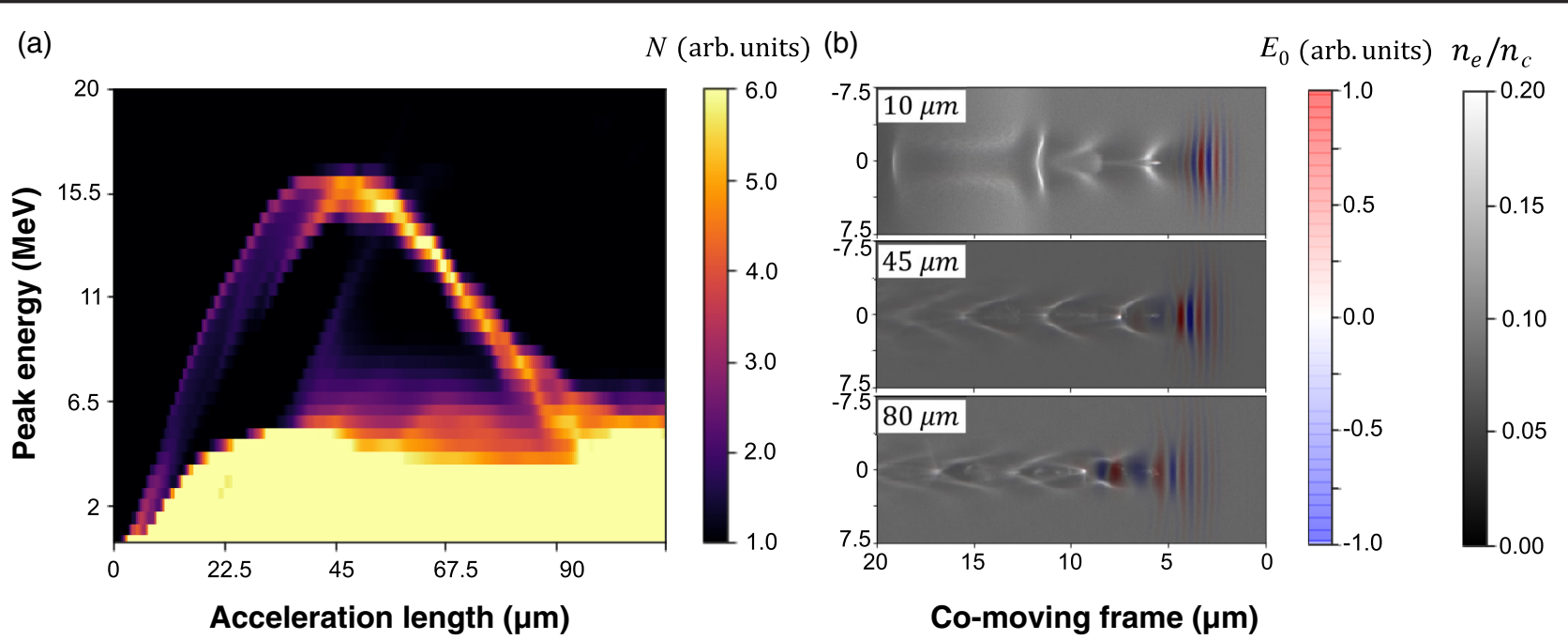

FIG. 7. (a) 3D PIC simulation of the energy evolution of an electron bunch in a wakefield with a background density of $n_{e}=$ $7 \times 10^{19} \mathrm{~cm}^{-3}$ driven by a 5-fs pulse with $a_{0}=2.25$ with a central wavelength of $800 \mathrm{~nm}$. (b) Density $n_{e}$ and transverse electric field $E_{0}$ evolution during the dephasing processes at different acceleration lengths.

pulse elongation, did not limit the electron energy. We notice also that PIC simulations seem to agree better at lower densities and intensities. Further discrepancies between the simulations and the sub-5-fs experimental results are attributed to the use of: (i) $800 \mathrm{~nm}$ as the simulated driver wavelength as well as (ii) Gaussian beams with an ideal $M^{2}$, instead of $740 \mathrm{~nm}$ and $M^{2}>2$, typical for flattop beams. Moreover, (iii) propagation effects in the experiments were reduced by moving the focal plane of the laser accordingly for each shock-front position and finally, (iv) an uncertainty in the electron density determination of up to $15 \%-20 \%$. A typical systematic difference is that while a relevant broadening occurs in the PIC simulations towards both the red and the blue sides of the laser spectrum after the interaction, experimentally only blueshift was observed.

\section{CONCLUSION}

In conclusion, a systematic and direct measurement of the dephasing effect in a laser wakefield accelerator has been performed utilizing shock-front injection and sub-10fs laser pulses. The evolution of various electron parameters during dephasing has been observed. Through a parabolic fit, we describe the electron energy evolution during dephasing in a weakly nonlinear LWFA scenario. Despite its simplicity, it provides good agreement with our experimental findings as well as with those from other groups obtained in the regime of the acceleration length being shorter than the dephasing length. Our study of the dephasing effect is of primary importance for (i) plasma accelerators since it provides the most accurate characterization of this fundamental energy limitation of LWFA in a single stage and (ii) optimization of electron acceleration in the near-single-cycle regime, where dispersion plays an important role [54,55]. Last but not least, using short laser pulses as delivered by LWS-20 allows the generation of stable sub-10 MeV quasimonoenergetic electron beams. This is a promising electron source for ultrafast electron diffraction [56,57] with sub-10-fs temporal resolution, which is still about an order of magnitude better than standard technology, once other challenges such as the large divergence and energy spread are overcome.

\section{ACKNOWLEDGMENTS}

This work is supported by DFG-Project Transregio TR18, The Munich Centre for Advanced Photonics (MAP) and by the Euratom research and training program 2018-2014 under Grant Agreement No. 633053 within the framework of the EUROfusion Consortium. L. V. acknowledges the support from Swedish Research Council (201605409 and 2019-02376). The authors acknowledge the use of computational resources provided by the Swedish National Infrastructure for Computing (SNIC).

[1] T. Tajima and J. M. Dawson, Laser Electron Accelerator, Phys. Rev. Lett. 43, 267 (1979).

[2] J. Faure, Y. Glinec, A. Pukhov, S. Kiselev, S. Gordienko, E. Lefebvre, J.-P. Rousseau, F. Burgy, and V. Malka, A laserplasma accelerator producing monoenergetic electron beams, Nature (London) 431, 541 (2004).

[3] C. G. R. Geddes, C. Toth, J. Van Tilborg, E. Esarey, C. B. Schroeder, D. Bruhwiler, C. Nieter, J. Cary, and W. P. Leemans, High-quality electron beams from a laser wakefield accelerator using plasma-channel guiding, Nature (London) 431, 538 (2004).

[4] S. P. D. Mangles, C. D. Murphy, Z. Najmudin, A. G. R. Thomas, J. L. Collier, A. E. Dangor, E. J. Divall, P. S. Foster, J. G. Gallacher, C. J. Hooker, D. A. Jaroszynski, 
A. J. Langley, W. B. Mori, P. A. Norreys, F. S. Tsung, R. Viskup, B. R. Walton, and K. Krushelnick, Monoenergetic beams of relativistic electrons from intense laser-plasma interactions, Nature (London) 431, 535 (2004).

[5] K. Schmid, L. Veisz, F. Tavella, S. Benavides, R. Tautz, D. Herrmann, A. Buck, B. Hidding, A. Marcinkevicius, U. Schramm, M. Geissler, J. Meyer-ter-Vehn, D. Habs, and F. Krausz, Few-Cycle Laser-Driven Electron Acceleration, Phys. Rev. Lett. 102, 124801 (2009).

[6] D. Guénot, D. Gustas, A. Vernier, B. Beaurepaire, F. Böhle, M. Bocoum, M. Lozano, A. Jullien, R. Lopez-Martens, A. Lifschitz, and J. Faure, Relativistic electron beams driven by KHz single-cycle light pulses, Nat. Photonics 11, 293 (2017).

[7] H. T. Kim, K. H. Pae, H. J. Cha, I. J. Kim, T. J. Yu, J. H. Sung, S. K. Lee, T. M. Jeong, and J. Lee, Enhancement of Electron Energy to the Multi-GeV Regime by a Dual-Stage Laser-Wakefield Accelerator Pumped by Petawatt Laser Pulses, Phys. Rev. Lett. 111, 165002 (2013).

[8] X. Wang et al., Quasimonoenergetic laser-plasma acceleration of electrons to $2 \mathrm{GeV}$, Nat. Commun. 4, 1988 (2013).

[9] A. J. Gonsalves et al., Petawatt Laser Guiding and Electron Beam Acceleration to $8 \mathrm{GeV}$ in a Laser-Heated Capillary Discharge Waveguide, Phys. Rev. Lett. 122, 084801 (2019).

[10] A. Buck, M. Nicolai, K. Schmid, C. M. S. Sears, A. Sävert, J. M. Mikhailova, F. Krausz, M. C. Kaluza, and L. Veisz, Real-time observation of laser-driven electron acceleration, Nat. Phys. 7, 543 (2011).

[11] O. Lundh, J. Lim, C. Rechatin, L. Ammoura, A. BenIsmaill, X. Davoine, G. Gallot, J. P. Goddet, E. Lefebvre, V. Malka, and J. Faure, Few femtosecond, few kiloampere electron bunch produced by a laser-plasma accelerator, Nat. Phys. 7, 219 (2011).

[12] W. Lu, M. Tzoufras, C. Joshi, F. S. Tsung, W. B. Mori, J. Vieira, R. A. Fonseca, and L. O. Silva, Generating multi$\mathrm{GeV}$ electron bunches using single stage laser wakefield acceleration in a 3D nonlinear regime, Phys. Rev. ST Accel. Beams 10, 061301 (2007).

[13] C. G. R. Geddes, C. Tóth, J. Van Tilborg, E. Esarey, C. B. Schroeder, D. Bruhwiler, C. Nieter, J. Cary, and W. P. Leemans, Production of high-quality electron bunches by dephasing and beam loading in channeled and unchanneled laser plasma accelerators, Phys. Plasmas 12, 056709 (2005).

[14] W. P. Leemans, B. Nagler, A. J. Gonsalves, C. Tóth, K. Nakamura, C. G. R. Geddes, E. Esarey, C. B. Schroeder, and S. M. Hooker, GeV electron beams from a centimeterscale accelerator, Nat. Phys. 2, 696 (2006).

[15] S. Karsch, J. Osterhoff, A. Popp, T. P. Rowlands-Rees, Z. Major, M. Fuchs, B. Marx, R. Hörlein, K. Schmid, L. Veisz, S. Becker, U. Schramm, B. Hidding, G. Pretzler, D. Habs, F. Grüner, F. Krausz, and S. M. Hooker, GeV-scale electron acceleration in a gas-filled capillary discharge waveguide, New J. Phys. 9, 415 (2007).

[16] S. P. D. Mangles, G. Genoud, M. S. Bloom, M. Burza, Z. Najmudin, A. Persson, K. Svensson, A. G. R. Thomas, and C. G. Wahlström, Self-injection threshold in self-guided laser wakefield accelerators, Phys. Rev. ST Accel. Beams 15, 011302 (2012).
[17] W. Rittershofer, C. B. Schroeder, E. Esarey, F. J. Grüner, and W. P. Leemans, Tapered plasma channels to phase-lock accelerating and focusing forces in laser-plasma accelerators, Phys. Plasmas 17, 063104 (2010).

[18] Q. Yu, Y. J. Gu, X. F. Li, S. Huang, F. Zhang, Q. Kong, Y. Y. Ma, and S. Kawata, Controllable high-quality electron beam generation by phase slippage effect in layered targets, Phys. Plasmas 21, 113106 (2014).

[19] E. Guillaume, A. Döpp, C. Thaury, K. Ta Phuoc, A. Lifschitz, G. Grittani, J.-P. Goddet, A. Tafzi, S. W. Chou, L. Veisz, and V. Malka, Electron Rephasing in a LaserWakefield Accelerator, Phys. Rev. Lett. 115, 155002 (2015).

[20] B. Hidding, G. G. Manahan, O. Karger, A. Knetsch, G. Wittig, D. A. Jaroszynski, Z.-M. Sheng, Y. Xi, A. Deng, J. B. Rosenzweig, G. Andonian, A. Murokh, G. Pretzler, D. L. Bruhwiler, and J. Smith, Ultrahigh brightness bunches from hybrid plasma accelerators as drivers of 5th generation light sources, J. Phys. B 47, 234010 (2014).

[21] A. Debus, R. Pausch, A. Huebl, K. Steiniger, T. E. Cowan, U. Schramm, and M. Bussmann, Circumventing the Dephasing and Depletion Limits of Laser-Wakefield Acceleration, Phys. Rev. X 9, 031044 (2019).

[22] A. Dubietis, G. Jonušauskas, and A. Piskarskas, Powerful femtosecond pulse generation by chirped and stretched pulse parametric amplification in BBO crystal, Opt. Commun. 88, 437 (1992).

[23] D. E. Rivas et al., Next generation driver for attosecond and laser-plasma physics, Sci. Rep. 7, 5224 (2017).

[24] D. Herrmann, L. Veisz, R. Tautz, F. Tavella, K. Schmid, V. Pervak, and F. Krausz, Generation of sub-three-cycle, 16 TW light pulses by using noncollinear optical parametric chirped-pulse amplification, Opt. Lett. 34, 2459 (2009).

[25] A. H. Zewail, 4D ultrafast electron diffraction, crystallography, and microscopy, Annu. Rev. Phys. Chem. 57, 65 (2006).

[26] G. Sciaini and R. J.D. Miller, Femtosecond electron diffraction: Heralding the era of atomically resolved dynamics, Rep. Prog. Phys. 74, 09610 (2011).

[27] J. B. Hastings, F. M. Rudakov, D. H. Dowell, J. F. Schmerge, J. D. Cardoza, J. M. Castro, S. M. Gierman, H. Loos, and P. M. Weber, Ultrafast time-resolved electron diffraction with megavolt electron beams, Appl. Phys. Lett. 89, 184109 (2006).

[28] J. Maxson, D. Cesar, G. Calmasini, A. Ody, P. Musumeci, and D. Alesini, Direct Measurement of sub-10 fs Relativistic Electron Beams with Ultralow Emittance, Phys. Rev. Lett. 118, 154802 (2017).

[29] E. C. Snively, M. A. K. Othman, M. Kozina, B. K. OforiOkai, S. P. Weathersby, S. Park, X. Shen, X. J. Wang, M. C. Hoffmann, R. K. Li, and E. A. Nanni, Femtosecond Compression Dynamics and Timing Jitter Suppression in a THzDriven Electron Bunch Compressor, Phys. Rev. Lett. 124, 054801 (2020).

[30] F. Qi, Z. Ma, L. Zhao, Y. Cheng, W. Jiang, C. Lu, T. Jiang, D. Qian, Z. Wang, W. Zhang, P. Zhu, and X. Zou, Breaking 50 Femtosecond Resolution Barrier in MeV Ultrafast Electron Diffraction with a Double Bend Achromat Compressor, Phys. Rev. Lett. 124, 134803 (2020). 
[31] K. Schmid, A. Buck, C. M. S. Sears, J. M. Mikhailova, R. Tautz, D. Herrmann, M. Geissler, F. Krausz, and L. Veisz, Density-transition based electron injector for laser driven wakefield accelerators, Phys. Rev. ST Accel. Beams 13, 091301 (2010).

[32] A. Buck, J. Wenz, J. Xu, K. Khrennikov, K. Schmid, M. Heigoldt, J. M. Mikhailova, M. Geissler, B. Shen, F. Krausz, S. Karsch, and L. Veisz, Shock-Front Injector for High-Quality Laser-Plasma Acceleration, Phys. Rev. Lett. 110, 185006 (2013).

[33] H.-E. Tsai, K. K. Swanson, S. K. Barber, R. Lehe, H.-S. Mao, D. E. Mittelberger, S. Steinke, K. Nakamura, J. van Tilborg, C. Schroeder, E. Esarey, C. G. R. Geddes, and W. Leemans, Control of quasimonoenergetic electron beams from laser-plasma accelerators with adjustable shock density profile, Phys. Plasmas 25, 043107 (2018).

[34] K. K. Swanson, H.-E. Tsai, S. K. Barber, R. Lehe, H.-S. Mao, S. Steinke, J. Van Tilborg, K. Nakamura, C. G. R. Geddes, C. B. Schroeder, E. Esarey, and W. P. Leemans, Control of tunable, monoenergetic laser-plasmaaccelerated electron beams using a shock-induced density downramp injector, Phys. Rev. Accel. Beams 20, 051301 (2017).

[35] S. P. D. Mangles, A. G. R. Thomas, O. Lundh, F. Lindau, M. C. Kaluza, A. Persson, C. G. Wahlström, K. Krushelnick, and Z. Najmudin, On the stability of laser wakefield electron accelerators in the monoenergetic regime, Phys. Plasmas 14, 056702 (2007).

[36] T. Kurz, J.P. Couperus, J. M. Krämer, H. Ding, S. Kuschel, A. Köhler, O. Zarini, D. Hollatz, D. Schinkel, R. D'Arcy, J.-P. Schwinkendorf, J. Osterhoff, A. Irman, U. Schramm, and S. Karsch, Calibration and cross-laboratory implementation of scintillating screens for electron bunch charge determination, Rev. Sci. Instrum. 89, 093303 (2018).

[37] S. Corde, C. Thaury, A. Lifschitz, G. Lambert, K. T. Phuoc, X. Davoine, R. Lehe, D. Douillet, A. Rousse, and V. Malka, Observation of longitudinal and transverse selfinjections in laser-plasma accelerators, Nat. Commun. 4, 1501 (2013).

[38] S. P. D. Mangles, A. G. R. Thomas, M. C. Kaluza, O. Lundh, F. Lindau, A. Persson, F. S. Tsung, Z. Najmudin, W. B. Mori, C. G. Wahlström, and K. Krushelnick, LaserWakefield Acceleration of Monoenergetic Electron Beams in the First Plasma-Wave Period, Phys. Rev. Lett. 96, 215001 (2006).

[39] E. Esarey, C. B. Schroeder, and W. P. Leemans, Physics of laser-driven plasma-based electron accelerators, Rev. Mod. Phys. 81, 1229 (2009).

[40] C. D. Decker and W. B. Mori, Group velocity of largeamplitude electromagnetic waves in a plasma, Phys. Rev. E 51, 1364 (1995).

[41] C. D. Decker, W. B. Mori, K.-C. Tzeng, and T. Katsouleas, The evolution of ultraintense, short-pulse lasers in underdense plasmas, Phys. Plasmas 3, 2047 (1996).
[42] L. M. Gorbunov, S. Y. Kalmykov, and P. Mora, Laser wakefield acceleration by petawatt ultrashort laser pulses, Phys. Plasmas 12, 033101 (2005).

[43] C. B. Schroeder, C. Benedetti, E. Esarey, and W. P. Leemans, Nonlinear Pulse Propagation and Phase Velocity of Laser-Driven Plasma Waves, Phys. Rev. Lett. 106, 135002 (2011).

[44] J. Faure, Y. Glinec, J. J. Santos, F. Ewald, J. P. Rousseau, S. Kiselev, A. Pukhov, T. Hosokai, and V. Malka, Observation of Laser-Pulse Shortening in Nonlinear Plasma Waves, Phys. Rev. Lett. 95, 205003 (2005).

[45] A. Popp, Dynamics of electron-acceleration in laser-driven wakefields: Acceleration limits and asymmetric plasma waves, $\mathrm{PhD}$ thesis, LMU, München, 2011, http://hdl .handle.net/11858/00-001M-0000-000F-ABEC-D.

[46] G. Sun, E. Ott, Y. C. Lee, and P. Guzdar, Self-focusing of short intense pulses in plasmas, Phys. Fluids 30, 526 (1987).

[47] W. Lu, C. Huang, M. Zhou, W. B. Mori, and T. Katsouleas, Nonlinear Theory for Relativistic Plasma Wakefields in the Blowout Regime, Phys. Rev. Lett. 96, 165002 (2006).

[48] O. Jansen, T. Tückmantel, and A. Pukhov, Scaling electron acceleration in the bubble regime for upcoming lasers, Eur. Phys. J. Special Topics 223, 1017 (2014).

[49] A. Pukhov and J. Meyer-ter-Vehn, Laser wakefield acceleration: The highly nonlinear broken-wave regime, Appl. Phys. B 74, 355 (2002).

[50] J. Götzfried, A. Döpp, M. F. Gilljohann, F. M. Foerster, H. Ding, S. Schindler, G. Schilling, A. Buck, L. Veisz, and S. Karsch, Physics of High-Charge Electron Beams in LaserPlasma Wakefields, Phys. Rev. X 10, 041015 (2020).

[51] A. Gonoskov, Ultra-intense laser-plasma interaction for applied and fundamental physics, $\mathrm{PhD}$ thesis, Umeå University, Umeå, 2014, http://urn.kb.se/resolve?urn=urn: nbn:se:umu:diva-84245.

[52] C. Nieter and J. R. Cary, VORPAL: A versatile plasma simulation code, J. Comput. Phys. 196, 448 (2004).

[53] S. Chou, J. Xu, K. Khrennikov, D. E. Cardenas, J. Wenz, M. Heigoldt, L. Hofmann, L. Veisz, and S. Karsch, Collective Deceleration of Laser-Driven Electron Bunches, Phys. Rev. Lett. 117, 144801 (2016).

[54] B. Beaurepaire, A. Lifschitz, and J. Faure, Electron acceleration in subrelativistic wakefields driven by fewcycle laser pulses, New J. Phys. 16, 023023 (2014).

[55] S. Y. Kalmykov, A. Beck, X. Davoine, E. Lefebvre, and B. A. Shadwick, Laser plasma acceleration with a negatively chirped pulse: All-optical control over dark current in the blowout regime, New J. Phys. 14, 033025 (2012).

[56] Z.-H. He, A. G. R. Thomas, B. Beaurepaire, J. A. Nees, B. Hou, V. Malka, K. Krushelnick, and J. Faure, Electron diffraction using ultrafast electron bunches from a laserwakefield accelerator at $\mathrm{kHz}$ repetition rate, Appl. Phys. Lett. 102, 064104 (2013).

[57] J. Faure, B. Van Der Geer, B. Beaurepaire, G. Gallé, A. Vernier, and A. Lifschitz, Concept of a laser-plasma-based electron source for sub-10-fs electron diffraction, Phys. Rev. Accel. Beams 19, 021302 (2016). 